Sección Historia del Pensamiento Jurídico 

Revista de Estudios Histórico-Jurídicos

[Sección historia del pensamiento jurídico]

XL (Valparaíso, Chile, 2018)

[pp. 407 - 421]

\title{
Thomas Hobbes y la FUnCión de LOS JUeCES*
}

[Thomas Hobbes and the Role of the Judges]

\author{
Carlos Isler Soto** \\ Universidad Bernardo O'Higgins, Santiago, Chile \\ Universidad Mayor, Santiago, Chile
}

\begin{abstract}
RESUMEN
En este artículo expondremos la visión de la función del juez que tiene Thomas Hobbes, mostrando que es bastante más sofisticada de lo que, a primera vista, podría deducirse de sus posiciones en materia de filosofía política, y de lo que parece desprenderse de su desacuerdo con Coke. Para ello, expondremos en primer lugar su disputa con Coke respecto al papel de los jueces y juristas en relación al derecho (I). A continuación, expondremos en qué consiste la función del juez según Hobbes
\end{abstract}

\begin{abstract}
This article presents Thomas Hobbes' view on the role of the judges, which is far more sophisticated than it may seem given his opinion on political philosophy matters and his disagreement with Coke. Consequently, we first set forth the controversy with Coke on the role of the judges regarding the law (I). Secondly, we present what the judge's role according to Hobbes is (II and III), his institutional position (IV), and finally, we will pose (V)
\end{abstract}

ReCibido el 25 de julio de 2017 y aCEPTADO el 30 de mayo de 2018

* Este artículo forma parte del proyecto Fondecyt de Iniciación 11150285, financiado por Conicyt, titulado "Los fundamentos filosóficos de la teoría del contrato en Thomas Hobbes", del cual el autor es investigador responsable.

** Licenciado en Ciencias Jurídicas y Sociales, Universidad Austral de Chile. Licenciado en Filosofía, Universidad de los Andes. Doctor en Derecho, Pontificia Universidad Católica de Chile. Profesor de Filosofía del Derecho en la Universidad Bernardo O'Higgins y de Introducción al Derecho en la Universidad Mayor. Dirección postal: Escuela de Derecho Universidad Bernardo O'Higgins, General Gana 1702, Santiago, Chile. Correo electrónico: carlos_isler@yahoo.com. 
(II y III), su posición institucional (IV), para terminar mostrando $(\mathrm{V})$ que su disputa con Coke se refiere, más que a la función del juez, al concepto mismo de derecho.

Palabras Clave

Thomas Hobbes - Edward Coke Jueces - Subsunción. that his controversy with Coke refers to the very concept of law rather than to the role of the judge.

KEYWORDS

Thomas Hobbes - Edward Coke Judges - Subsumption.

\section{La CRÍTICA De HobBes a COKE}

La doctrina hobbesiana acerca de la naturaleza de la función judicial ha recibido escasa atención por parte de los estudiosos ${ }^{1}$. Hobbes expone su doctrina de la función judicial en gran medida comentando las ideas del juez Edward Coke. Cuando se expone la teoría del derecho de Hobbes, es usual resaltar su disputa con el juez Coke con la que inicia su conocido Dialogue, aunque sin mencionar la relevancia que tiene ello para determinar la naturaleza de la función judicial. Uno de los pasajes que más ha llamado la atención de los estudiosos es aquél en el cual Hobbes refuta la doctrina de Coke respecto a la relación entre derecho y razón ${ }^{2}$. Ambos están de acuerdo respecto de que el derecho encarna la razón, pero difieren respecto de qué razón encarna. Coke había expresado que "la razón es la vida del derecho, es más, el common law mismo no es sino razón; lo que debe entenderse como una perfección artificial de la razón, obtenida mediante extenso estudio, observación, y experiencia, y no como la razón natural de todo hombre; pues, Nemo nascitur artifex. Esta razón jurídica (legall reason) est summa ratio y consiguientemente, si toda la razón que se encuentra dispersa en tantas múltiples cabezas, fuese unida en una sola, todavía no podría hacer un derecho tal como es el derecho en Inglaterra; porque a través de muchas sucesiones de épocas ha sido ajustado y refinado por un número infinito de hombres serios y eruditos, y por la larga experiencia ha llegado a tal perfección para el gobierno de este reino, que en

1 Sólo hay dos artículos que tratan o se refieren al tema Campagna, Nobert, Leviathan and its Judges, en Archiv für Rechts- und Sozialphilosophie, 86/4 (2000), pp. 499-517, y DyzENHAUs, David, The Very Idea of a Judge, en The University of Toronto Law Journal, 60/1 (2010), pp. 61-80. Campagna expone que "The very few pages of Leviathan dedicated to judges belong, unfortunately, to the most neglected pages of Hobbes's political and juridical philosophy": p. 500. Curiosamente, en su artículo solo se concentra en lo que Hobbes expone sobre los jueces en el Leviathan, dejando de lado el Dialogue, que para este tema es mucho más importante. Por eso resulta, nuevamente, extraño que exprese que "it is only in Leviathan that the question of the role of judges in a State is discussed in some detail': p. 514.

2 Así, comentan este conocido párrafo, entre otros, Martin Loughlin en The political jurisprudence of Thomas Hobbes, en Dyzenhaus, David; Poole, Thomas (eds.), Hobbes and the Law (New York, Cambridge University Press, 2012), pp. 10-11; Thomas Poole en Hobbes on law and prerogative, en Dyzenhaus; Poole (eds.), cit., pp. 77-78; Luc Foisneau, en Hobbes. La vie inquiète (Paris, Gallimard, 2016), pp. 284-285, y Martin Kriele, en Notes on the Controversy between Hobbes and English Jurists, en KoselLeCK, Reinhart; SCHnur, Roman (eds.), Hobbes-Forschungen (Berlin, Duncker \& Humblot, 1969), pp. 212-214. 
él puede con justicia verificarse la antigua regla, Neminem esse oportet sapientorem legibus: nadie debiera ser a partir de su razón privada más sabio que el derecho, el cual es la perfección de la razón"3.

Hobbes cita en dos ocasiones este pasaje en su Dialogue y se refiere a él una vez en el Leviathan, y lo hace siempre para criticar la identificación que hace Coke entre el derecho y la razón de los jueces y juristas. Una doctrina como la de Coke reviste a jueces y juristas de una facultad que no les corresponde, la de crear derecho, y las consecuencias de una doctrina como la de Coke serían la anarquía o la guerra civil. Ya al comenzar el Dialogue, el personaje Filósofo expresa que "[...] Que la razón que es la vida del derecho deba ser no natural, sino artificial, no puedo concebirlo. Entiendo lo suficientemente bien que el conocimiento del derecho se obtiene por mucho estudio, tal como lo son todas las otras ciencias, las cuales cuando son estudiadas y obtenidas, lo son por la razón natural y no por la razón artificial. Te concedo que el conocimiento del derecho es un arte, pero no que cualquier arte de algún hombre, o de muchos, por muy sabios que sean, o el trabajo de uno y más artífices, por muy perfecto que sea, sea el Derecho. No es la sabiduría, sino la autoridad la que crea el derecho. Son también oscuras las palabras razón jurídica; no hay razón en las criaturas terrestres sino la razón humana; pero supongo que quiere decir que la razón de un juez, o de todos los jueces juntos (sin el rey) es tal Summa Ratio, y el derecho mismo, lo que niego, porque nadie puede hacer un derecho sino aquel que tiene el Poder Legislativo. Que el derecho haya sido ajustado por hombres serios y eruditos, queriendo decir los profesores de derecho, es manifiestamente falso, pues todas las leyes de Inglaterra han sido hechas por los reyes de Inglaterra, consultando a la nobleza y a los comunes en el Parlamento, de los cuales ni siquiera uno entre veinte era un abogado erudito.

Ab. Tú hablas del derecho legislado (Statute Law), y yo hablo del Common Law.

Fil. Hablo del Derecho en general"4.

Del mismo modo, en el diálogo II, que trata sobre la soberanía, vuelve a criticar la doctrina de Coke. El personaje Filósofo dice que: "Aunque sea verdad que ningún hombre nazca con el uso de razón, sin embargo, todos los hombres pueden llegar a ejercitarla tan bien como los abogados; y cuando han aplicado su razón a las leyes (las cuales eran leyes antes de que las estudiaran, o si no no era el derecho lo que estudiaron) pueden ser tan idóneos y capaces de la judicatura como Sir. Edw. Coke mismo, quien no era juez porque tuviese más o menos uso de razón, sino porque el rey lo hizo tal: y mientras dice que un hombre que tuviese tanta razón como se encuentra dispersa en tantas cabezas no podría hacer un derecho tal como es este derecho de Inglaterra; si uno le preguntase ¿quién creó el derecho de Inglaterra? ¿Diría él que una sucesión de abogados o jueces Ingleses

${ }^{3}$ COKE, Edward, The First Part of the Institutes of the Laws of England; or, a Commentary upon Littleton (New York, The Lawbook Exchange, 1999), I, sect 138, $97 \mathrm{~b}$.

${ }^{4}$ Hobbes, Thomas, A Dialogue between a Philosopher \& a Student of the Common Laws of England (Chicago, The University of Chicago Press, 1971), I, 55 (en adelante, "D”: se citará mencionando primero en cuál diálogo se encuentra el texto, y luego la página de la edición ocupada. Así, "D I, 55”, significa que el texto citado se encuentra en el primer diálogo y en la página 55 de la edición de The University of Chicago Press). 
lo crearon, o más bien una sucesión de reyes; y que basados en su propia razón, sea en solitario, o con el Consejo de los Lords y Comunes en el Parlamento, sin los jueces, u otros profesores de derecho? Ves entonces que la razón del rey, sea ésta mayor o menor, es aquella Anima Legis, aquella Summa Lex, de la cual habla Sir Edw. Coke, y no la razón, erudición, o sabiduría de los jueces; pero puedes ver que a través de sus Institutes of Law repetidas veces aprovecha de magnificar la erudición de los abogados, a quienes llama siempre los sabios del parlamento, o del consejo del rey"s.

Por lo tanto, para Thomas Hobbes, a diferencia de Coke, los jueces no crean derecho, y sólo deben aplicarlo. Pareciera desprenderse de ello, en consecuencia, que fueren meran máquinas aplicadoras de normas a hechos, tal como pretendo el formalismo jurídico.

La impresión anterior se acentúa si recordamos la visión general que tiene de la razón Thomas Hobbes: la razón es cálculo, y su perfección la alcanza en la ciencia, la cual es un conjunto de teoremas deducidos rigurosamente por medios puramente lógicos a partir de ciertas premisas indubitables. El modelo de razonamiento es el matemático: "Cuando un hombre razona, no hace sino concebir una suma total, de la a d i c i ó n de parcelas; o concebir un remanente, de la s u s t r a c c i ó n de una suma respecto de otra: lo cual (si es hecho mediante palabras) es concebir las consecuencias a partir de los nombres de todas las partes al nombre del todo; o de los nombres del todo y una parte, al nombre de la otra parte" ${ }^{6}$. Y este modelo de razonamiento calculador se aplica incluso al derecho: "Pues como los aritméticos enseñan a sumar y restar en $\mathrm{n}$ ú m e r o s [...] Los escritores de políticos suman juntos pactos para encontrar los d e beres de los hombres; y los abogados, leyes, yhechos, para encontrar lo que es correcto eincorrecto en las acciones de hombres privados. En suma, en tantas materias como hay lugar para la a d i c i ó n y s u s t r a c c i ó $n$, hay también lugar para la r a z ó n; y donde aquéllas no tienen lugar, ahí la r a z ó n no tiene nada que hacer" 7 .

Una ciencia del derecho, como la que él pretende que puede deducirse de su

5 D II, 62. Por otro lado, ya en el Leviathan había expresado que “[...] the doubt is, of whose Reason it is, thath shall be received for Law. It is not meant of any private Reason; for then there would be as much contradiction in the Lawes, as there is in the Schooles; nor yet, (as Sr. Ed. Coke makes it,) an 'Artificiall perfection of Reason, gotten by long study, observation, and experience', (as his was.) For it is possible long study may encrease, and confirm erroneous Sentences: and where men build on false grounds, the more they build, the greater is the ruine: and of those that study, and observe with equall time, and diligence, the reasons and resolutions are, and must remain, discordant: and therefore it is not that 'Jurisprudentia', or wisedome of subordinate Judges; but the Reason of this our Artificiall Man the Common-wealth, and his Command, that maketh Law: and the Common-wealth being in their Representative but one Person, there cannot easily arise any contradiction in the Lawes; and where they doth, the same Reason is able, by interpretation or alteration, to take it away": HobBes, Thomas, Leviathan (Oxford, Oxford University Press, 2012) XXVI, 422 (en adelante, " $L$ "; se citará indicando primero el capítulo y luego la página en la que se encuentra el texto; así, "L XXVI, 422", significa que el texto citado se encuentra en el capítulo XXVI, página 422 de la edición ocupada). La version Latina del Leviathan expone el mismo argumento, pero sin la referencia a Coke. Véase L XXVI, 423.

${ }^{6} \mathrm{~L} \mathrm{~V}, 64$.

${ }^{7} \mathrm{LV}, 64$. 
filosofía política, debe tener la misma forma que la geometría, y sus conclusiones deben ser rigurosamente deducidas desde las premisas. La función judicial es, entonces, ante todo subsunción de un hecho bajo normas, y es precisamente eso lo que afirma: se trata de sumar normas y hechos, para obtener una conclusión. Nótese cómo encontramos aquí en Hobbes claramente la idea del silogismo jurídico, idea cuyo origen se atribuye normalmente a Beccaria.

Por otro lado, Hobbes afirma una dependencia estricta de los jueces respecto del soberano. Más aún: el juez actúa siempre como delegado del soberano, aquel que detenta el poder político. Hobbes no acepta la separación de poderes, ya que cree que ningún Estado puede subsistir si sus poderes se encuentran divididos. La división de poderes lleva, inevitablemente, piensa Hobbes, a la guerra civil. De este modo, el legislador necesariamente es, a la vez, juez supremo. Así puede decir que "en todas las controversias, la judicatura pertenece originalmente al rey", en "todas las cortes de justicia, el soberano (quien es la persona de la república), es quien juzga", y que "también aquellos a quienes se les otorga jurisdicción, son ministros públicos. Pues en las sedes de la justicia representan la persona del soberano; y la sentencia de ellos, es su sentencia" ${ }^{10}$.

Por ende, tanto la filosofía política de Hobbes, que ve al juez como esencialmente subordinado al legislador (no hay separación de poderes), sin capacidad para crear derecho; su desacuerdo respecto de la relación entre derecho y juez con Coke; y su teoría general del razonamiento, derivada de premisas antropológicas más generales, parecieran implicar que la función del juez fuese la mecánica de la subsunción mediante un silogismo jurídico, vale decir, la misma que se atribuye al formalismo jurídico en general, y que tan criticada ha sido por la teoría del derecho contemporánea por ser de modo evidente descriptivamente falsa. Y, sin embargo, existen pasajes en los que Hobbes reconoce que la función del juez no se limita a la mera subsunción, debiendo, en ocasiones, ejercer razonamiento teleológico e histórico para determinar cuál sea la premisa mayor del silogismo jurídico, vale decir, para ejercer el razonamiento jurídico en su plenitud.

\section{INTERPRETACIÓN DE LA LEY}

Ante todo, lejos de cualquier formalismo ingenuo, Hobbes reconoce que hay leyes cuya interpretación es dudosa ${ }^{11}$. Del mismo modo, tiene claro que siendo el tenor literal el primer elemento de interpretación de las mismas, no puede ser el único. De este modo, expresa que el juez puede "sin peligro apartarse de la letra [de la ley], si no lo hace del significado y sentido de la ley, el cual puede ser fácilmente encontrado por un hombre sabio (tal como lo son comúnmente los jueces) por

${ }^{8}$ D III, 77.

${ }^{9}$ L XXVI, 422.

${ }^{10}$ L XXIII, 380.

${ }^{11}$ Cfr., por ejemplo, D III, 83: "the Quarrels arising about the Lands of Religious Persons cannot arise from the Lands, but from the doubfulness of the Laws". Igualmente, L XXVI, 436: "For the signification of almost all words, are either in themselves, or in the metaphorical use of them, ambiguous; and may be drawn in argument, to make many senses; but there is only one sense of the Law". 
el preámbulo, la época cuando fue hecha, y los inconvenientes por los cuales fue hecha"12. Lo que importa es determinar, en tales casos, el fin que tuvo el legislador al momento de dictar la ley: "no es siempre el derecho lo que es significado por la interpretación g r a m a t i c a l de la letra, sino lo que el legislador entendía que a través de ella debería tener vigor; intención que, confieso, es cosa muy difícil en muchas ocasiones de captar a partir de las palabras de la ley, y requiere una gran capacidad de entendimiento, y mayores meditaciones, y consideraciones de tal coyuntura de ocasiones, e incomodidades tales que necesitaban una nueva ley como remedio" ${ }^{3}$. Del mismo modo, en el Leviathan expone que "el juez subordinado debe atender a la razón que movió a su soberano a hacer tal ley, de modo que su sentencia esté de acuerdo con ello; la cual es entonces la sentencia de su soberano; de otro modo, es suya propia, y una injusta" ${ }^{14}$.

Ahora bien, este razonamiento teleológico que intenta determinar el fin de las leyes particulares se realiza teniendo siempre en consideración el fin de la ley en general: "el alcance de toda ley humana es la paz, y justicia en cada nación entre ellos mismos, y defensa contra enemigos externos" 15 . Tal es, precisamente, el fin del Estado, de modo que ninguna ley particular puede tener algún fin específico que sea incompatible con este objetivo general. De este modo, la teoría política de Hobbes determina también su teoría de la interpretación.

Del mismo modo, corresponde al juez interpretar la ley natural, que rige en caso de silencio de las leyes positivas: "La interpretación de la ley de la naturaleza, es la sentencia del juez constituido por la autoridad soberana, para oír y determinar tales controversias que dependan de ella, y consiste en la aplicación de la ley al caso actual"16.

Más aun: lejos, nuevamente, de cualquier tipo de formalismo, Hobbes reconoce que los jueces deben resolver, en ocasiones, casos que la ley, según su tenor literal, no cubre. Y es que el legislador no puede preverlo todo. En tales casos, para fallar el juez debe realizar el mismo razonamiento teleológico que al interpretar la ley: descubierto el fin de la normativa vigente, puede determinar si cubre el caso específico o no. Y si no, debe él mismo proveer la solución, aplicando la equidad ${ }^{17}$. Y, en palabras que podría haber suscrito Aristóteles, el personaje Filósofo expresa que "Concluyo que la justicia completa la ley, y la equidad interpreta la ley; y corrige los juicios dados en base a la misma ley: en lo cual no me aparto mucho de

${ }^{12}$ D I, 56. Sin embargo, tal apartarse de la letra de la ley debe hacerse con prudencia, de lo contrario, se transforman en oscuras leyes que no lo son de suyo: así el personaje Filósofo dice que "For my part I believe that Men at this day have better learn't the Art of Caviling against the Words of a Statute, tan heretofore they had, and thereby encourage themselves, and others, to undertake Suits upon little reason": D III, 84.

13 D III, 97-98.

${ }^{14}$ L XXVI, 422.

15 D II, 57.

${ }^{16}$ L XXVI, 430.

17 "for there be a very great many reasonable Exceptions almost to every General Rule, which the makers of the Rule could not foresee; and very many Words in every Statute, especially long ones, that are, as to Grammar, of Ambiguous signification, and yet to them that know well, to what end the Statute was made, perspicuous enough": D III, 98. 
la definición de equidad, citada por Sir Edw. Coke, I Inst. Sect. 21. viz.: la equidad es una cierta razón perfecta que interpreta y corrige la ley escrita"18.

Lo que sí Hobbes comparte con el formalismo es un claro optimismo respecto de la posibilidad de dominar en poco tiempo y con facilidad el derecho legislado: "si pretendo dentro de un mes, o dos, volverme capaz de ejecutar el oficio de juez, no has de pensar que sea arrogancia; pues debes concederme a mí, tanto como a otros hombres, mi pretensión de razón, la cual es el Common Law (recuerda esto de modo que no deba hacértelo presente nuevamente, que la razón es el Common Law) y en lo relativo al derecho legislado (Statute Law), viendo que se encuentra impreso, y que hay índices que me dirijan a cualquier materia contenida en ellos, pienso que un hombre puede sacar mucho provecho de ellos en dos meses"19.

Tras realizar este razonamiento teleológico e histórico, el juez llega a fijar un sentido a la ley, transformándose de este modo, en cuanto delegado del soberano, en intérprete auténtico de ellas. Esto es particularmente interesante, porque Hobbes, en su crítica a Coke, había rechazado la idea de que el juez fuese creador del derecho. Pero negándole tal carácter, le reconoce el de intérprete auténtico del mismo, al menos respecto de las partes del juicio: "del mismo modo, cuando la cuestión es sobre el significado de leyes escritas, no es intérprete de ellas, aquel que escribe un comentario sobre ellas. Pues los comentarios se encuentran normalmente más sujetos a disputa que el texto; y consiguientemente necesitan otros comentarios; y de este modo no habrá fin para tal interpretación. Y consiguientemente, si no existe un intérprete autorizado por el soberano, del cual no hayan de separarse los jueces subordinados, el intérprete no puede ser otro que los jueces ordinarios, del mismo modo a como lo son en casos de la ley no escrita; y sus sentencias deben ser consideradas por aquellos que litigan, por leyes en tal caso particular; pero no han de vincular a otros jueces a dictar sentencias similares en casos similares. Pues un juez puede errar incluso en la interpretación de leyes escritas; pero ningún error de un juez subordinado puede cambiar la ley, la cual es la sentencia general del soberano" ${ }^{20}$.

Pudiese parecer contradictorio negar carácter creador de derecho a los jueces, y reconocerles, al mismo tiempo, el carácter de intérpretes auténticos del mismo. Sin embargo, no hay tal contradicción, ya que al negar carácter creador de derecho a la actividad judicial lo que Hobbes quiere hacer es expresar el imperativo de que los jueces deben adecuarse a una realidad normativa preexistente al fallar, de modo que su actividad no es libre, y de modo que también pueden equivocarse al intentar realizar dicha adecuación. Reconocer carácter de intérprete auténtico del derecho no equivale a negar la posibilidad de decisiones judiciales erróneas, ya que él mismo reconoce que los jueces pueden equivocarse. Quiere, más bien, expresar la necesidad de que las partes del juicio traten la sentencia judicial como si ésta fuese una interpretación correcta de la ley, aun cuando en los hechos no lo sea. Tal doctrina se funda, nuevamente, en su teoría política más general: sólo

\footnotetext{
${ }^{18}$ D III, 101.

19 D I, 56.

${ }^{20}$ L XXVI, 436.
} 
procediendo de esta manera es posible la paz y la seguridad, fines supremos del Estado $^{21}$.

Pero la sentencia que obliga a las partes, precisamente por no ser el juez creador de derecho, no obliga a los jueces posteriores, y puede ser revertida por estos o por el soberano. Todo lo anterior va mostrando la importancia que tienen los jueces en el pensamiento de Hobbes: en cuanto delegados del soberano son agentes de paz.

Un juez, se decía, puede equivocarse al interpretar una ley ${ }^{22}$ (aun cuando las partes del juicio deban considerar su interpretación como auténtica). Y es que un buen juez, aquel que no se equivoca, requiere ciertas cualidades, y aquí es donde Hobbes muestra de modo más claro que está lejos de concebir al juez como una mera máquina: "las cosas que hacen a un buen juez, o un buen intérprete de las leyes, son, primero, un a correct a comprensión de aquella ley de la naturaleza principal llamada e q u i d a d; la que no dependiendo de la lectura de los escritos de otros hombres, sino de la bondad de la razón natural de un hombre y meditación, se presume que existe sobre todo en aquellos que han tenido más ocio (leisure), y tuvieron la mayor inclinación a meditar sobre ello. En segundo lugar, desprecio de riquezas innecesarias, y privilegios. En tercer lugar, ser capaz en el juicio de desprenderse de todo miedo, enojo, odio, amor, y compasión. En cuarto lugar, y finalmente, paciencia para escuchar; atención diligente en el escuchar; y memoria para retener, asimilar, y aplicar lo que ha escuchado"23.

${ }^{21}$ Como expresa Campagna: "An interpretation is to be accounted authentic if it corresponds to the true meaning of the law. An interpretation is to be accounted authoritative if it creates a duty, i.e. if it is binding... when Hobbes considers the relationship judge-subject, he presupposes the notion of an authoritative interpretation, but when he considers the relationship judge-judge, he presupposes the notion of an authentic interpretation. The subject, in other words, has to accept the authoritative interpretation as the authentic interpretation": CAMPAGNA, Norbert, cit. (n. 1), p. 506.

22 "And though a Judge should (as all Men may do) Erre in his Judgment [...]", D III, 98. Igualmente cfr. L XXVI, 432: "But because there is no Judge Subordinate, nor Soveraign, but may erre in a Judgment of Equity [...]”.

${ }^{23}$ L XXVI, 438. Por otro lado, como dice Campagna, "notice that in order to be an authoritative interpreter of the laws one does not need to be a good interpreter": CAMPAGNA, Norbert, cit. (n. 1), p. 510. La exigencia de desprenderse de miedo, enojo, odio, amor y compasión no debe entenderse como que el juez debe ser desapasionado, tal como lo malinterpretan Gross, James J. y Maroney, Terry A., The Ideal of the Dispassionate Judge: An Emotion Regulation Perspective, en Emotion Review, 6/2 (2014), p. 142. Tras citar este pasaje del Leviathan, sostienen que Hobbes habría sido el paradigma del defensor del ideal del juez desapasionado, " a legal standing ideal" que "stands in sharp conflict with the reality of flesh-and-blood judges" (p. 142), dado que "emtionless judges are mythical beings" (p. 144). Lejos de Hobbes defender que los jueces deban carecer de emociones: para el filósofo de Malmesbury, las pasiones son necesariamente móvil -al menos parcial- de toda acción humana, de modo que un ser humano desapasionado no podría actuar. Cfr. el capítulo VI del Leviathan, en el cual trata de las pasiones como móvil de actuación, y hace un catastro de ellas, enumerando muchas cuya ocurrencia no es incompatible con el ejercicio de la función judicial. Lo que Hobbes exige es que el juez se desprenda de las particulares pasiones que menciona. Con todo, debe aclararse que, cuando Hobbes dice que el juez debe desprenderse de todo amor, no puede querer decir con a $\mathrm{m}$ o r y o d i o aquello que ambos términos significan en el sentido técnico que da al término en el capítulo VI: "That which 


\section{INTERPRETACIÓN DE LOS CONTRATOS}

Del mismo modo que las leyes, y por la misma razón, los contratos deben interpretarse teleológicamente. Aquí Hobbes se enfrenta al conocido problema de los términos implícitos en un contrato. Ellos deben descubrirse atendiendo al fin que han tenido las partes al contratar. Por ello, "si un hombre por palabras, u otros signos, parece despojarse del fin, para el cual dichos signos fueron establecidos; no debe entenderse como si lo hubiese significado, o que era su voluntad; sino que era ignorante respecto de cómo tales palabras y acciones habrían de ser entendidas" ${ }^{24}$.

Este principio sirve para determinar los derechos no especificados por las partes en el contrato: atendido el fin de contrato, se determinan cuáles fueron los derechos transferidos, y una vez determinados estos, se determinan cualesquiera otros derechos transferidos en atención a su carácter de medios respecto de los primeros. Así, “el que transfiere cualquier derecho, transfiere los medios de disfrutarlo, tanto como esté en su poder. Como el que vende terreno, se entiende que transfiere el pastizal, y cualquier cosa que crezca sobre él; ni puede aquél que vende un molino desviar la corriente que lo mueve" 25 .

La exigencia de tener en vista el fin de las partes al contratar deriva del egoísmo sicológico que sostiene Hobbes. En efecto, para el filósofo de Malmesbury toda acción humana tiene por necesidad como fin algún bien para el agente. Así lo expresa en todas sus obras políticas ${ }^{26}$.

\section{LA POSICIÓN INSTITUCIONAL DEL JUEZ}

Hobbes niega al juez la capacidad de crear derecho, aun cuando su visión de la función judicial sea muy distinta de la del formalismo que normalmente va asociado a la negación de la doctrina de que el juez cree derecho.

Ante todo, debe recalcarse que el juez se encuentra estrictamente sometido a la ley, sin poder cambiarla ni dejar de aplicarla ${ }^{27}$. La posibilidad de que el juez ejerza

men Desire, they are also sayd to LOVE: and to HATE those things, for which they have Aversion. So that Desire, and Love, are the same thing; save that by Desire, we always signifie the Absence of the Object; by Love, most commonly the Presence of the same. So also by Aversion, we signifie the Absence; and by Hate, the Presence of the Object": L VI, 80.

${ }^{24} \mathrm{~L}$ XIV, 202.

${ }^{25} \mathrm{~L}$ XIV, 210.

${ }^{26}$ Por ejemplo, en Elements of Laws (Oxford, Oxford University Press, 2008), XIV, 78-79: "Necessity of nature maketh men to will and desire 'bonum sibi', that which is good for themselves"; De Cive (Oxford, Oxford University Press, 1983) I, XIII, 96-97: “nam vnusquisque naturali necessitate bonum sibi appetit", o L, XXV, 400: "[...] to gain some other Good to himselfe; which (as I said before) is of every mans will the proper object”. Sobre el egoísmo sicológico en Hobbes en general, cfr. Zarka, Yves, La Décision métaphysique de Hobbes. Conditions de la politique (Paris, Vrin, 1999), y sobre la relación del egoísmo sicológico con su teoría jurídica, véase IsLER, Carlos, El iusnaturalismo egoísta de Thomas Hobbes (Madrid, Marcial Pons, 2017), especialmente pp. 67-74.

${ }^{27}$ Si el $A$ Discourse of Laws es de Hobbes, entonces habría afirmado ya en una época muy temprana esta necesidad de sujeción a la ley: "The dispensers and interpreters of the Law, be the Magistrates and Judges, and all sorts and degrees of men whatsoever be tied and bound to the observance of the same. To this purpose, Solon being demanded, 'What City was best governed?' 
razonamiento teleológico o aplique la equidad no significa que pueda dejar de aplicar la ley una vez determinado su sentido o su rango de aplicación. Y Hobbes cree que dicha sujeción del juez a la ley sólo se puede garantizar si se entiende al juez como delegado del soberano, vale decir, del legislador. No puede haber separación de poderes, ya que un juez independiente del soberano es un juez no sujeto la ley. Sujeción a la ley significa sujeción al legislador.

Precisamente por ser un delegado del soberano, el juez está estrictamente obligado a aplicar la ley. No puede controlarla. "Viendo que todas las leyes, escritas y no escritas, tienen su autoridad, y fuerza, de la voluntad de la república; es decir, de la voluntad del representante [...] un hombre puede admirarse de dónde proceden tales opiniones, como se encuentran en los libros de abogados de eminencia en muchas repúblicas, directamente o por consecuencia haciendo al Poder Legislativo depender de hombres privados, o jueces subordinados" 28 . No puede haber control judicial de las leyes: aunque en ocasiones el juez debe aplicar directamente la ley natural, ello ocurre en silencio de la ley. Pero cuando el sentido de la ley es claro, atendido el fin del legislador, debe aplicarse siempre: "de modo que lo incomodidad que sigue a las meras palabras de una ley escrita, puedan llevarlo [al juez] a la intención de la ley, mediante la cual interpretarla mejor; pero ninguna Incomodidad puede justificar una Sentencia contra la Ley. Pues cada juez de lo correcto e incorrecto, no es juez de lo que es beneficioso o incómodo para la república" ${ }^{29}$. Del mismo modo, la obligación de buscar la intención del legislador al aplicar la ley se explica por el carácter del juez de delegado del soberano ${ }^{30}$.

Es este carácter de delegado del soberano el que explica, por otra parte, una de las doctrinas más novedosas de Hobbes: que la costumbre jurídica también sea mandato del soberano. En efecto, para Hobbes todo el derecho positivo emana del soberano, y ello incluye la costumbre, la cual es un mandato tácito del soberano -ya que este es superior del juez, y al ver que el juez la aplica, y pudiendo

answered, 'That, wherein the City obeyed the Magistrates, and the Magistrates the Laws". HoBBEs, Thomas, A Discourse of Laws, en ÉL MISMO, Three Discourses, ReYNOLDS, Noel y SAXONHOUSE, Arlene (eds.) (Chicago, University of Chicago Press, 1995), p. 111. Sin embargo, como se sabe, la atribución a Hobbes de tal obra y, en general, de cualquiera de los tres discursos encontrados en Horae Subsecivae, es materia de discusión. Por ejemplo, John Fortier niega tal atribución en Hobbes and 'A Discourse of Laws': The Perils of Wordprint Analysis, en The Review of Politics, 59/4 (1997), pp. 861-888. Por otro lado, parece aceptarla James Murphy, en The Philosophy of Positive Law. Foundations of Jurisprudence (New Haven and London, Yale University Press, 2005), Kindle Edition, pos. 3481. Los editores de la obra de Hobbes defienden su atribución y responden a Fortier en Hilton, John; Reynolds, Noel y SaXonhouse, Arlene, Hobbes and 'A Discourse of Laws': Response to Fortier, The Review of Politics, 59/4 (1997), pp. 889-903.

${ }^{28}$ L XXVI, 420.

${ }^{29}$ L XXVI, 438.

${ }^{30}$ De ahí que no concordemos con Foisneau cuando dice que cuando Hobbes exige al juez buscar la intención del legislador, "Le problème n'est pas, en l'ocurrence, d'assurer la soumission des juges au pouvoir exécutif, mais de fournir à ces derniers un principe de jugement conforme au principe de souverainetê" : FOISNEAU, Luc, cit. (n. 2), p. 285. Por supuesto que no se trata de asegurar la sumisión del juez al poder ejecutivo, sino al soberano, el cual es el legislador. Recordemos que en Hobbes no hay separación de poderes. 
impedirlo, no lo hace, debe entenderse que manifiesta su aprobación-: "Cuando el largo uso adquiere la autoridad de una ley, no es la extensión del tiempo lo que crea la autoridad, sino la voluntad del soberano significada por su silencio (pues el silencio es a veces prueba del consentimiento)" 31 . De este modo, aun cuando Hobbes reconozca que con anterioridad a su aplicación por el juez la costumbre carezca de carácter jurídico, en ningún caso ello le lleva a reconocer carácter creador del derecho al juez en cuanto tal: quien crea derecho siempre es el soberano, aun cuando lo haga mediante el juez, su delegado.

\section{El sentido de la CRÍtica de Hobbes a CoKe}

Se ha visto que para Hobbes los jueces no son meras máquinas aplicadoras de leyes a hechos, como suponen los diversos formalismos. Hobbes podría concordar con Coke respecto de que el juez debe ejercitar razonamiento teleológico e histórico para interpretar leyes y contratos, que en ocasiones debe aplicar la equidad, que la ciencia del derecho que poseen los jueces requiere estudio -aunque considere que el estudio requerido para dominar tal ciencia no es tan exigente, ya que se puede dominar la materia en dos meses-, que su interpretación del derecho es auténtica, y que un buen juez requiere tener ciertas cualidades. Más aún, concordaría con Coke respecto de la importancia de buenos jueces para el adecuado funcionamiento del Estado. De ahí que pueda decir que "los Jueces merecen el honor y beneficio que disfrutan"32. Además, ellos son el equivalente a los órganos de la voz del cuerpo político $^{33}$, ya que ellos expresan al resto de la comunidad cuál sea el derecho ${ }^{34}$.

${ }^{31}$ L XXVI, 416. Esta doctrina será, como es sabido, retomada por John Austin, quien expresamente afirma haberla obtenido de Hobbes. Cfr. Austin, John The Province of Jurisprudence Determined, en ÉL MISMO, The Province of Jurisprudence Determined and The Uses of the Study of Jurisprudence (Indianapolis, Hackett, 1998), pp. 31-32. Tal doctrina será, a su vez, duramente criticada por H.L.A. Hart, quien sostendrá que no puede necesariamente interpretarse la inacción de un superior respecto de las acciones de un inferior como muestra de satisfacción ante dicha acción. Cfr. HART, H.L.A., The Concept of Law (2a ed., New York, Oxford University Press, 1994), pp. 46-47.

${ }^{32}$ D III, 98.

33 "These publique persons, with Authority from the Soveraign Power, either to Instruct, or Judge the people, are such members of the Common-wealth, as may fitly be compared to the organs of Voice in a Body Naturall': L XXIII, 382.

${ }^{34}$ Sin embargo, esto no debe entenderse, como lo hace Campagna, como si en ausencia de un pronunciamiento judicial el derecho no fuese vinculante: "But if the nature of law consists in the authentic interpretation of the legislator's meaning, and if the judge's sentence constitutes, or is always to be taken as constituting the authentic interpretation of the legislator's meaning, it is the judge who constitutes law as an obligatory command out of the words of the sovereign. The law, we could also say, unfolds its obligatoriness only through the judge's sentence": CAMPAGNA, Norbert, cit. (n. 1), p. 507. Lo anterior no puede ser correcto porque, primero, Hobbes expresa que, además de los jueces, son órganos de la voz del cuerpo político los ministros encargados de instruir al pueblo. Así, por ejemplo, dice que "unlesse there be an Interpreter authorised by the Soveraign, from which the subordinate Judges are not to recede, the Interpreter can be no other than the ordinary Judges [...]": L XXVI, 436. En segundo lugar, una doctrina que sostuviese que, antes de la aplicación por parte de un juez, una norma no es obligatoria, es claramente falsa, ya que implica que los jueces no aplican las normas por ser obligatorias, sino que las aplican a pesar de que todavía no 
Pues Bien: si hay tantas concordancias entre Hobbes y Coke, ¿por qué critica tan duramente su definición de derecho? La diferencia entre Hobbes y Coke no es sobre la naturaleza de la función judicial, sino sobre la relación entre el concepto de derecho y los jueces. La definición de derecho de Coke hace de los jueces los creadores de este, y ello es lo que Hobbes considera errado y peligroso. El concepto de derecho de Hobbes es normativista, ya que concibe al derecho como un conjunto de normas, el conjunto de los mandatos del soberano. Sólo un tal concepto, piensa Hobbes, puede dar cuenta de la necesidad irrestricta del juez de someterse a la ley. Por eso dice que los jueces y juristas, al adquirir la ciencia del derecho, se adecúan a una realidad normativa preexistente, una realidad que ellos no pueden cambiar a su voluntad. Una definición de derecho como la de Coke, piensa Hobbes, que hace a los jueces creadores de derecho, es peligrosa porque da lugar a la idea de que el Poder Judicial sea independiente del soberano, y puede legitimar la no aplicación por parte de un juez de la ley escrita. Y es que, para Hobbes, el juez siempre debe aplicar la ley ${ }^{35}$.

Hobbes nunca menciona el caso Bonham, aquel caso de 1610 en el cual el juez Coke se negó a aplicar una ley aprobada por el Rey en el Parlamento, aduciendo que dicha ley atentaba contra la razón ${ }^{36}$, pero es claro que tenía una tal realidad en mente al criticar a Coke: como expresa Stoner, "la independencia de la judicatura del common law respecto del canciller o incluso del rey, que Coke parece defender en algunos aspectos, es insoportable" ${ }^{37}$ para Hobbes. La definición de derecho de Coke implica, cree Hobbes, la idea de independencia judicial y revisión judicial de las leyes, y ambas ideas son nefastas y generadoras de división en el cuerpo político. De hecho, Hobbes cree que una de las causas de la Guerra Civil

lo son. Por ejemplo, implicaría que, antes de ser aplicada por un juez, una ley que estableciese como límite máximo de velocidad en carretera de $140 \mathrm{~km} / \mathrm{h}$ no sería obligatoria. Cfr. lo que dice Hart sobre la teoría del mandato tácito: "there is very little plausibility in the view that a statute made by a past 'sovereign' is not law until it is actually applied by the courts in the particular case, and enforced with the acquiescence of the present sovereign. If this theory is right it follows that the courts do not enforce it because it is already law [...]": HART, H. L. A., cit. (n. 31), p. 64. Más aún, la doctrina es absurda porque el mismo hecho de que las partes comparezcan ante un juez supone que ellas ya han captado como obligatorias las normas jurídicas que constituyen a una persona en juez, y ello con anterioridad a cualquier pronunciamiento de tal persona. Siendo, por ende, dicha doctrina absurda, no conviene, a falta de texto expreso, atribuirla a un gran filósofo.

35 "It cannot be that a Written Law should be against Reason: For nothing is more reasonable than that every Man should obey the Law, which he hath himself assented to": D III, 97.

${ }^{36}$ Como se sabe, según la interpretación tradicional, el juez Coke habría declarado inválida una ley por oponerse al Common Law, por lo que este caso sería uno de los antecedentes de la teoría del control judicial de las leyes. Un exponente de dicha interpretación tradicional es George P. Smith en Dr. Bonham's Case and the Modern Significance of Lord Coke's influence, en Washignton Law Review, 41 (1966), quien llama a Coke “legal father of judicial review”, p. 297. Una interpretación distinta del caso Bonham, según la cual Coke, más que invalidar una ley, pretendía hacer una interpretación estricta de la misma, en DORADO PORRAs, Javier, La lucha por la Constitución. Las teorias del Fundamental Law en la Inglaterra del siglo XVII, (Madrid, CEPC, 2001), pp. 75-80; y en Helmholz, R. H., Bonham's Case, Judicial Review, and the Law of Nature, en Journal of Legal Analysis, 1/1 (2009), pp. 325-354.

${ }^{37}$ Stoner, James, Common Law \& Liberal Theory. Coke, Hobbes, \& the Origins of American Constitutionalism (Kansas, University Press of Kansas, 1992), p. 118. 
inglesa fue la adopción de la teoría de separación de poderes por parte de jueces y juristas, y la consiguiente pretensión de que los jueces, y el Parlamento como judicatura suprema, podían actuar con independencia del legislativo (que Hobbes consideraba era el rey $)^{38}$. Las definiciones de derecho no son inocentes, sino que tienen consecuencias prácticas, y ello explica la animadversión de Hobbes por $\mathrm{Coke}^{39}$, no obstante los múltiples puntos de acuerdo que, según se vio, pudiesen tener respecto de la naturaleza de la función judicial.

Todo lo anterior explica el que Hobbes sostenga que, aunque las partes quedan obligadas por la interpretación que de la ley hace el juez, la sentencia de un juez no obligue a los jueces posteriores. Porque si el juez no es creador de derecho, sino que debe adecuarse a una realidad preexistente, puede suceder que un juez se equivoque al interpretar o aplicar el derecho, que preexiste a la decisión judicial, y la obligación del juez posterior es adecuar su decisión a dicha realidad normativa preexistente a la decisión del primer juez, el derecho como conjunto de normas, y por eso el segundo juez puede y debe apartarse de la decisión del primer juez.

${ }^{38}$ Cfr. los siguientes párrafos de Behemoth, su conocido diálogo sobre la Guerra Civil inglesa: "A [...] Vpon this the Parliament Vote a Standing to their former Votes, as also that when the Lords and Commons in Parliament, which is the Supreame Court of Judicature in the Kigndome shall declare what the Law of the Land is, to haue this not onely questioned but contradicted is a high breach of the Priuiledge of Parliament. B. I thought that he that makes the Law ought to declare what the Law is, for what is else to make a Law, but to declare what it is?": HoBBES, Thomas, Behemoth (Oxford, Oxford University Press, 2010) II, p. 241. Aquí el personaje A expone la pretension del Parlamento que, como Tribumal Supremo, sostenía que su interpretación del derecho podía prevalecer incluso frente a la del rey, y el personaje B le responde que, con ello, le quitan al rey su poder legislativo, desde que la suprema judicatura es anexa al poder legislativo. Hobbes cree que esta doctrina de separación de poderes e independencia judicial, que se manifestaría también en la definición de derecho de Coke, fue una de las causas de la guerra civil. "For the Lawyers, I mean the Judges of the Courts at Westminster and some few others, though but Auocates, yet of great reputation for their skill in the Common Laws and Statutes of England, had infected most of the Gentry of England with their Maximes and Cases praeiudged, which they call Presidents, and made them thinke so well of their owne knowledge in the Law, that they were very glad of this occasion to shew it against the King": Behemoth, III, p. 266. Sobre la doctrina de la separación de poderes o soberanía dividida como origen, en el pensamiento de Hobbes, de la guerra civil inglesa, véase JENDRYSIK, Mark Stephen, Explaining the English Revolution. Hobbes and his Contemporaries (Lanham, Lexington Books, 2002), pp. 121-133.

${ }^{39}$ Hobbes expresa en repetidas ocasiones una constante animadversion por Coke. Así, por ejemplo, dice que Coke con sus opiniones deseaba aumentar su poder político: D III, 88-89 "I cannot believe that Sir Edw, Coke, how much soever he desir'd to advance the authority oh himself, and other Justices of the Common-Law, could mean that the King in the King's Bench sate as a Spectator only"; comete falacias en sus razonamientos: "Sir Edw. Coke as he had (you see) in many places before, hath put a Fallacy upon himself, D III, 89; sus argumentos no tienen valor, "For that Argument of Sir Edw. Coke's, that the King has given away all his Judicial Power, is worth nothing" (D III, 90). Respecto de otro de sus argumentos, dice que "This is not an Argument worthy of the meanest Lawyer" (D IV, 105). Coke, por otro lado, fuerza a las leyes para hacerlas decir lo que él quiere que digan: "This Statute cannot be by Sir Edw. Cokes Torture made to say it" (D VI, 135). Y, en general, "Truly I never read weaker reasoning in any Author of the Law of England, than in Sir Edw. Coke's Institutes" (D VII, 156-157). Por todo lo anterior, puede decir James Stoner que "In the text of Hobbes's Dialogue it becomes clear beyond a doubt that the target of the work, from start to finish, is sir Edward Coke": STONER, James, cit. (n. 37), p. 118. 
Una definición de derecho como la de Coke que hacía de los jueces creadores de derecho, parece implicar la idea de que los jueces fuesen infalibles. La idea de que exista el error judicial sólo es posible si se entiende al derecho como un conjunto de normas y no como el resultado de la actividad de los jueces.

\section{BiBLIOGRAFÍA}

Austin, John, The Province of Jurisprudence Determined [1832], en ÉL MISMO, The Province of Jurisprudence Determined and The Uses of the Study of Jurisprudence (Indianapolis, Hacket, 1998).

Campagna, Norbert, Leviathan and its Judges, en Archiv für Rechts- und Sozialphilosophie, 86/4 (2000), pp. 499-517.

COKE, Edward, The First Part of the Institutes of the Laws of England; or, a Commentary upon Littleton (18 ${ }^{\mathrm{a}}$ ed., 1823, reimpresión New York, The Lawbook Exchange, 1999).

Dyzenhaus, David, The Very Idea of a Judge, en The University of Toronto Law Journal, 60/1 (2010), pp. 61-80.

Dorado Porras, Javier, La lucha por la Constitución. Las teorias del Fundamental Law en la Inglaterra del siglo XVII (Madrid, CEPC, 2001).

Foisneau, Luc, Hobbes. La vie inquiète (Paris, Gallimard, 2016).

FORTIER, Jon, Hobbes and 'A Discourse of Laws': The Perils of Wordprint Analysis, en The Review of Politics, 59/4 (1997), pp. 861-888.

Gross, James J. y Maroney, Terry A., The Ideal of the Dispassionate Judge: An Emotion Regulation Perspective, en Emotion Review, 6/2 (2014), pp. 142-151.

Hart, H.L.A., The Concept of Law (2a ed., New York, Oxford University Press, 1994).

Helmholz, R. H., Bonham's Case, Judicial Review and the Law of Nature, en Journal of Legal Analysis, 1/1 (2009), pp. 325-354.

Hilton, John; Reynolds, Noel; y Saxonhouse, Arlene, Hobbes and "A Discourse of Laws": Response to Fortier, en The Review of Politics, 59/4 (1997), pp. 889-904.

Hobbes, Thomas, A Dialogue between a Philosopher and a Student of the Common Laws of England [1681] (ed. por Joseph Cropsey, Chicago, The University of Chicago Press, 1971).

- Behemoth or the Long Parliament [1681] (ed. por Paul Seaward, Oxford, Oxford University Press, 2010). Vol. 10 de la edición crítica Clarendon de las obras de Thomas Hobbes.

- De Cive [1642] (ed. por Howard Warrender, Oxford, Oxford University Press, 1983). Vol. 2 de la edición crítica Clarendon de las obras de Thomas Hobbes.

- The Elements of Law. Human Nature and De Corpore Politico [1640] (ed. por J.C.A. Gaskin, Oxford, Oxford University Press, 2008).

— Leviathan [1651 y 1668] (ed. por Noel Malcolm, Oxford, Oxford University Press, 2012). Vols. 3, 4 y 5 de la edición crítica Clarendon de las obras de Thomas Hobbes.

- A Discourse of Laws [1620], en ÉL MISMO, Three Discourses (ed. por Reynolds, Noel y Saxonhouse, Arlene, Chicago, University of Chicago Press, 1995).

IsLER, Carlos, El iusnaturalismo egoista de Thomas Hobbes (Madrid, Marcial Pons, 2017). JendrysiK, Mark Stephen, Explaining the English Revolution. Hobbes and his Contemporaries (Lanham, Lexington Books, 2002).

KrIELE, Martin, Notes on the Controversy between Hobbes and English Jurists, en KosE- 
LLECK, Reinhart y SCHNuR, Roman (eds.), Hobbes-Forschungen (Berlin, Duncker \& Humblot, 1969), pp. 211-222.

Loughlin, Martin, The political jurisprudence of Thomas Hobbes, en DyzenHaus, David y Poole, Thomas (eds.), Hobbes and the Law (New York, Cambridge University Press, 2012), pp. 5-21.

Murphy, James, The Philosophy of Positive Law. Foundations of Jurisprudence, (New Haven and London, Yale University Press, 2005), Kindle Edition.

Poole, Thomas, Hobbes on law and prerogative, en Dyzenhaus, David y Poole, Thomas (eds.), Hobbes and the Law (New York, Cambridge University Press, 2012), pp. 68-96.

Smith, Stephen P, Dr. Bonham's Case and the Modern Significance of Lord Coke's Influence, en Washington Law Review, 41 (1966), pp. 297-314.

StOner, James, Common Law \& Liberal Theory: Coke, Hobbes and the Origins of American Constitutionalism (Kansas, University Press of Kansas, 1992).

ZarKa, Yves Charles, La Décision métaphysique de Hobbes. Conditions de la politique (Paris, Vrin, 1999). 
